\title{
Influence of laser photocoagulation for clinically significant diabetic macular oedema (DMO) on short-wavelength and conventional automated perimetry
}

\author{
C. Hudson ${ }^{1,2}$, J. G. Flanagan ${ }^{1,3}$, G. S. Turner ${ }^{1}$, H. C. Chen ${ }^{1}$, L. B. Young ${ }^{1}$, D. McLeod ${ }^{1}$ \\ ${ }^{1}$ University Department of Ophthalmology, Manchester Royal Eye Hospital, Manchester, UK \\ ${ }^{2}$ School of Biomedical Sciences, University of Ulster, Coleraine, Northern Ireland \\ ${ }^{3}$ Department of Ophthalmology, University of Toronto, The Toronto Hospital, Toronto, Ontario, Canada
}

\begin{abstract}
Summary The aim of the study was to determine the effect of laser photocoagulation for clinically significant diabetic macular oedema (DMO) on macular visual function as assessed by conventional and shortwavelength automated static threshold perimetry. The sample comprised 24 patients who required laser photocoagulation for clinically significant DMO (mean age 59.75 years, range $45-75$ years). One eye of each patient was selected for the study. Patients underwent conventional and short-wavelength perimetry using programme 10-2 of the Humphrey Field Analyser on two separate occasions prior to treatment and subsequently within 1 week of, and at 1,2 , 4 and 12 weeks after, treatment. The pointwise pattern deviation plot was analysed for conventional perimetry and a pointwise horizontal and vertical hemifield asymmetry analysis was derived for shortwavelength perimetry (thereby negating the influence of pre-receptoral absorption). The extent of sensitivity loss was determined by counting the number of stimulus locations with statistical probability levels of $p$ less than 0.05. Group mean log minimum angle of resolution ( $\log$ MAR) visual acuity was largely un-
\end{abstract}

changed over the course of the study. Conventional perimetry showed an increase in the group mean number of abnormal contiguous stimulus locations from 2.4 (SD 4.3, range 0-14) immediately prior to treatment, to 12.4 (SD 7.8, range 0-30) within 1 week of treatment; at 3 months post-treatment, the group mean number of abnormal contiguous stimulus locations was 8.1 (SD 6.5, range 0-20). A similar but less pronounced change was found for short-wavelength perimetry. The spatial position of the posttreatment localised sensitivity loss corresponded with the area of retinal photocoagulation. Despite proven benefit in the stabilisation of visual acuity, laser photocoagulation for clinically significant DMO invariably results in a localised loss of perimetric sensitivity within $10^{\circ}$ eccentricity of the fovea. Evidence for the value of laser therapy for clinically significant DMO must be re-examined. [Diabetologia (1998) 41: 1283-1292]

Keywords Automated perimetry, visual field loss, laser photocoagulation, diabetic macular oedema.
Macular oedema is the leading cause of visual impairment and legal blindness in diabetic patients [1,2].

Received: 20 April 1998 and in revised form: 22 June 1998

Corresponding author: Dr. C. Hudson, Manchester Royal Eye Hospital University Dept. of Ophthalmology, Oxford Road, Manchester M13 9WH, UK

Abbreviations: SWS, Short-wavelength sensitive; DMO, diabetic macular oedema; logMAR, log minimum angle of resolution; LOCS, Lens Opacities Classification System; HFA, Humphrey Field Analyser; SWAP, short-wavelength automated perimetry.
Diabetic macular oedema (DMO) is considered treatable; guidelines have been set for the initiation of laser therapy and a role for laser photocoagulation has been established following clinical trials [3-7]. Clinically significant DMO represents the point at which the initiation of laser therapy is recommended irrespective of visual acuity since central vision is 'threatened'. Clinically significant DMO is defined as one or more of the following [8]; (i) thickening of the retina at or within 500 microns of the centre of the macula; (ii) hard exudates at or within $500 \mathrm{mi}$ crons of the centre of the macula, if associated with 
thickening of the adjacent retina; and (iii) retinal thickening one disc diameter in size which is located within one disc diameter of the centre of the macula. Laser photocoagulation for clinically significant DMO results in the stabilisation, and occasionally the improvement, of visual acuity when compared with untreated control eyes [4-7]. A post-treatment reduction in the magnitude and extent of retinal thickening has been clinically observed by stereo fundus biomicroscopy and stereo photography [9-10].

In addition to stabilising visual acuity, grid laser photocoagulation of clinically significant DMO has also been suggested to benefit reading owing to increased parafoveal vision and improved scanning ability [11]. Other studies, however, have reported the occurrence of paracentral scotomata following grid laser photocoagulation for DMO [9-10]. Conversely, Striph and co-workers [12] documented a reduction in the mean sensitivity of Humphrey Field Analyser (HFA) programme 10-2 (using stimulus size I or III) in 64 eyes of 36 patients with DMO following grid laser photocoagulation (using an argon green, or krypton red, laser). Mean sensitivity within $5^{\circ}$ eccentricity of the fovea deteriorated by approximately 3.5 decibels $(\mathrm{dB})(\mathrm{SD} 3.8 \mathrm{~dB})$ as a result of the initial treatment session. Surprisingly, the shape of scotomata (i.e. originally resulting from the DMO) did not change following treatment, although the overall field exhibited a diffuse depression of sensitivity. The full effect of laser photocoagulation for clinically significant DMO on the central visual field is unclear.

The clinical challenge of undertaking laser photocoagulation for clinically significant DMO is to reconcile the benefits of treatment with potential sideeffects and complications, including foveal burn, exacerbation of pre-existing DMO, enlarging atrophic laser scars [13], subretinal neovascularisation and fibrosis [14] and paracentral scotomata [9-10]. It is generally accepted that laser photocoagulation for clinically significant DMO results in "visual compromise" [15]; a clearer understanding of the effect of laser photocoagulation on the central visual field needs to be gained before the true overall benefit of treatment can be judged. In a related study [16], we have shown that short-wavelength automated perimetry (SWAP) offers greater sensitivity for the detection of abnormality than conventional automated perimetry in patients with untreated clinically significant DMO. This paper details the effect of grid laser photocoagulation treatment on the central visual field in the same cohort of patients. The aim of the study was to determine the effect of laser photocoagulation for clinically significant DMO on macular visual function as assessed by log minimum angle of resolution (logMAR) visual acuity and by both conventional and short-wavelength perimetry.

\section{Material and methods}

Sample. The sample comprised 24 patients (18 men and 6 women) with clinically significant DMO as defined by the ETDRS criteria [8] of mean age 59.75 years (range 45-75 years); all 24 patients exhibited retinal thickening within 500 microns of the centre of the macula. The mean time from diagnosis of diabetes was 8.5 years (range 2-18 years); 3 were insulin-dependent and 21 were non-insulin dependent and receiving oral hypoglycaemic medication. One eye was randomly assigned to the study if both eyes exhibited clinically significant DMO; 11 right eyes and 13 left eyes were assessed. The precise demographic details of the sample, and the sample size estimation, have been described in a related publication [16].

Prior to inclusion of the patient in the study, two medical retina specialists were required to independently confirm the diagnosis of clinically significant DMO using contact, or Volk, lens fundus biomicroscopy and thereby agree to the necessity for laser photocoagulation. Stereo photography (to document the presence of DMO) and fluorescein angiography (to exclude patients with ischaemic maculopathy, document the degree of capillary leakage and identify cystoid macular oedema) were also undertaken on entry to the study. Other inclusion criteria for the diabetic patients comprised a visual acuity (i.e. $\log$ minimum angle of resolution, $\log$ MAR) of 0.25 or better using the $96 \%$ contrast Regan chart (i. e. Snellen equivalent visual acuity of $6 / 9$ to 6/12). Exclusion criteria included: (i) a distance refractive error greater than \pm 6.00 dioptres sphere, or \pm 1.50 dioptres cylinder or both; (ii) a family history of glauco$\mathrm{ma}$ in a first degree relative; (iii) an intra-ocular pressure of $22 \mathrm{mmHg}$ or more; (iv) any other eye disease or disorder (including lenticular opacity - see below); (v) any previous laser photocoagulation treatment; (vi) proliferative diabetic retinopathy and its sequelae, for example, vitreous haemorrhage and retinal detachment; (vii) central nervous system (CNS) disorders or psychiatric illness; and (viii) systemic medication with known CNS effects, for example tranquillisers. Lenticular opacity was graded on the basis of slit lamp appearance and the Lens Opacities Classification System (LOCS) III [17]. Eyes with the following LOCS III grades were excluded from the study: (i) nuclear colour more than NC2; (ii) nuclear opalescence more than NO2; (iii) cortical cataract more than $\mathrm{C} 2$; and (iv) posterior subcapsular cataract $\mathrm{P} 1$ or more.

Procedures. The refraction was checked at each session prior to the assessment of visual acuity and perimetry to compensate for any transient refractive changes associated with diabetes [18]. LogMAR visual acuity was assessed using the $96 \%$ Weber contrast Regan chart. The chart was illuminated to $100 \mathrm{cdm}^{-2}$ and the recommended viewing distance of $3 \mathrm{~m}$ was used. A by-letter scoring system was used that gave credit to each correct letter [19]. LogMAR visual acuity was assessed on two separate occasions prior to laser photocoagulation and the results of the second session were taken as baseline to minimise any learning effects. In addition, the $96 \%$ Weber contrast Regan chart was presented randomly with the 25 and $11 \% \mathrm{Re}-$ gan charts; this randomisation of chart presentation was designed to minimise any familiarity the patient may gain of the letter composition of the charts.

Perimetry is the assessment of visual sensitivity at retinal locations eccentric to the fovea. A HFA 640 (Humphrey Instruments Inc., San Leandro, Calif.; USA), the clinical "gold standard" automated static threshold projection perimeter, was used for this study. The method of static stimulus presentation and the variables used for conventional (white-on-white) and short-wavelength perimetry are detailed elsewhere [16]. 
Table 1. Group mean test time (with standard deviation in parenthesis) to complete each stimulus condition as a function of order for visits 2,3 , and 7

\begin{tabular}{|c|c|c|c|c|}
\hline & \multicolumn{2}{|c|}{ Conventional } & \multicolumn{2}{|c|}{ Short-Wavelength } \\
\hline & \multicolumn{2}{|c|}{$\begin{array}{l}1 \mathrm{st} \quad 2 \mathrm{nd} \\
\text { (minutes, seconds) }\end{array}$} & \multicolumn{2}{|c|}{$\begin{array}{l}\text { 1st } \\
\text { (minutes, seconds) }\end{array}$} \\
\hline Visit 2 & $\begin{array}{l}12,30 \\
(0,45)\end{array}$ & $\begin{array}{l}12,00 \\
(0,45)\end{array}$ & $\begin{array}{l}15,00 \\
(1,47)\end{array}$ & $\begin{array}{l}15,47 \\
(2,13)\end{array}$ \\
\hline Visit 3 & $\begin{array}{l}14,10 \\
(1,16)\end{array}$ & $\begin{array}{l}13,18 \\
(1,24)\end{array}$ & $\begin{array}{l}16,30 \\
(2,15)\end{array}$ & $\begin{array}{l}16,25 \\
(1,57)\end{array}$ \\
\hline Visit 7 & $\begin{array}{l}12,40 \\
(1,02)\end{array}$ & $\begin{array}{l}13,15 \\
(1,16)\end{array}$ & $\begin{array}{l}15,53 \\
(1,43)\end{array}$ & $\begin{array}{l}15,21 \\
(1,44)\end{array}$ \\
\hline
\end{tabular}

Table 2. Details of argon green $(514 \mathrm{~nm})$ laser photocoagulation

\begin{tabular}{rcllr}
\hline Patient & $\begin{array}{l}\text { Spot size } \\
\text { (microns) }\end{array}$ & $\begin{array}{l}\text { Duration } \\
\text { (seconds) }\end{array}$ & $\begin{array}{l}\text { Power } \\
\text { (Watts) }\end{array}$ & $\begin{array}{c}\text { No. of } \\
\text { ablations }\end{array}$ \\
\hline 1 & 100 & 0.10 & 0.35 & 40 \\
2 & 100 & 0.10 & 0.10 & 220 \\
3 & 50 & 0.20 & 0.30 & 43 \\
4 & 100 & 0.10 & 0.10 & 180 \\
5 & 50 & 0.10 & 0.40 & 99 \\
6 & 100 & 0.10 & 0.25 & 57 \\
7 & 100 & 0.10 & 0.20 & 18 \\
8 & 100 & 0.10 & 0.09 & 26 \\
9 & 100 & 0.10 & 0.12 & 33 \\
10 & 100 & 0.10 & 0.30 & 44 \\
11 & 100 & 0.10 & 0.20 & 90 \\
12 & 100 & 0.10 & 0.25 & 55 \\
13 & 100 & 0.10 & 0.07 & 180 \\
14 & 100 & 0.10 & 0.30 & 13 \\
15 & $100 / 200$ & 0.15 & 0.10 & 21 \\
16 & 100 & 0.10 & $0.14 / 0.18$ & 309 \\
17 & 50 & $0.10 / 0.25$ & 0.20 & 33 \\
18 & 100 & 0.10 & 0.25 & 36 \\
19 & 100 & 0.10 & 0.1 & 156 \\
20 & 100 & 0.10 & 0.25 & 100 \\
21 & 100 & 0.10 & 0.30 & 21 \\
22 & 100 & 0.10 & 0.25 & 87 \\
23 & 100 & 0.10 & 0.20 & 80 \\
24 & 100 & 0.10 & 0.30 & 58 \\
\hline
\end{tabular}

Conventional perimetry results in the mediation of information along various neural pathways but short-wavelength perimetry utilises stimulus variables that are thought to be mediated along a specific neural pathway i.e. the blue-yellow colour opponent channel [20]. Short-wavelength perimetry uses a relatively narrow band (cf. the polychromatic stimulus of conventional perimetry) blue stimulus to preferentially stimulate the short-wavelength sensitive pathway and a high luminance yellow background to saturate both the medium-wavelength and long-wavelength sensitive pathways and to simultaneously suppress rod activity [21, 22]. The group mean times to complete each stimulus condition as a function of order for visits 2, 3 and 7 are shown in Table 1.

Patients underwent perimetry using programme 10-2 of the Humphrey Field Analyser which comprised 69 stimulus locations within $10^{\circ}$ eccentricity of the fovea (including foveal threshold); this programme uses a square grid of stimulus locations which straddle the mid-lines with a $2^{\circ}$ inter-stimulus spacing. The distance refractive correction of the study eye, to- gether with the appropriate near addition for the patient's age, was used to correct for a viewing distance of $33 \mathrm{~cm}$.

Grid laser photocoagulation for clinically significant DMO. Prior to laser photocoagulation, the two medical retina specialists mapped the extent of DMO onto a clear acetate sheet which detailed the retinal vascular pattern of each eye (i. e. derived from a $20^{\circ} \times 20^{\circ}$ scanning laser tomography image). The mapping of the extent of DMO was always undertaken following perimetry. Each medical retina specialist was blind to the other's assessment of the extent of DMO and to the perimetry results. The level of agreement of the baseline mapping of the extent of DMO between the medical retina specialists was high [16].

All diabetic patients underwent a single session of argon green $(514 \mathrm{~nm})$ grid laser photocoagulation. The sample represented a wide range of treatment requirements. Precise details of the treatment for each patient, including (i) ablation spot size (range 50-200 microns); (ii) duration of ablation (range $0.10-0.25 \mathrm{~s}$ ) (iii) power setting (range $0.07-0.40 \mathrm{~W}$ ); and (iv) number of ablations (range 13-309) are detailed in Table 2. Care was taken to ensure that laser burns were not placed at the margin of, or within, the foveal avascular zone.

Visits. Perimetry was undertaken on two separate occasions using both conventional and short-wavelength parameters prior to laser photocoagulation. The results of the second perimetry session were taken as baseline to minimise the influence of learning [23]. The order of stimulus condition (i.e. conventional or short-wavelength) at visit 1 was varied between patients and was then systematically varied between visits (i.e. short-wavelength undertaken 1 st at visit 1,2 nd at visit 2 , 1 st at visit 3, etc.). For visit 2, 15 diabetic patients underwent shortwavelength perimetry first and 9 patients initially underwent conventional perimetry. Perimetry was then repeated within 1 week of, and at 1, 2, 4 and 12 weeks after, treatment. Mandatory rest periods were given at least every 5 min during each perimetry session to minimise the influence of fatigue [24]. The results from the first (mean 5 days after, range 3-10 days) and final (mean 12 weeks after, range 10-15 weeks) post-treatment sessions relative to baseline are presented since these illustrate the observed overall effect of laser photocoagulation for clinically significant DMO on macular visual function. The mapping of the extent of DMO was repeated for each post-treatment visit by a single medical retina specialist with a second independent assessment being undertaken at visits 3 or 4 and 7 (these results are outside the scope of this paper). In addition, stereo photography was undertaken for each post-treatment visit and fluorescein angiography was repeated on exit from the study.

Analysis. Fields that resulted in a false-positive or false-negative response rate greater than $33 \%$ and those that exhibited fixation losses greater than $20 \%$ (i.e. Heijl-Krakau technique [25]) were excluded from the analysis. The respective perimetry results from the patients were compared with databases of normal fields. For conventional perimetry, the established age-matched STATPAC II database [26] of HFA programme 10-2 was used. For short-wavelength perimetry, normal HFA programme 10-2 sensitivity values were derived from the results of 400 normal subjects between the ages of 18 and 84 years (mean age 48 years, SD 17) who had undergone short-wavelength perimetry using programme $30-2$ i.e. the HFA STATPAC for SWAP database. Of the subjects 230 were aged 18-50 years, 90 were 51-65 years and 80 were 66-84 years. A weighted linear interpolation procedure based upon the angular distance between the 10-2 stimulus location to be derived and the 4 surrounding 30-2 locations was used. 
The details of the interpolation procedure have been described in a related publication [16]. Normal subjects were recruited at various international centres for the HFA STATPAC II and the STATPAC for SWAP databases using equivalent inclusion and exclusion criteria as those used for the diabetic patients.

An analytical methodology was adopted for both perimetry conditions that recognised variation in the shape of the visual field relative to that of a normal database rather than depending upon absolute sensitivity values. For conventional perimetry, the pattern deviation plot of the HFA STATPAC II database was used to illustrate the pointwise deviation (i.e. of each stimulus location) of the shape of the visual field from the age-matched normal field [27, 28]. For short-wavelength perimetry, a pointwise horizontal hemifield asymmetry analysis was established. By this analytical approach, the influence of pre-receptoral absorption [29-32] and light scatter [33] was negated since the overall effect of these factors results in a symmetrical attenuation of the "hill of vision"; this is of particular relevance in diabetic patients who are known to exhibit exaggerated ocular media absorption values [34]. A pointwise vertical hemifield asymmetry analysis was also undertaken in order to show the full extent of any localised field loss [16]. The derivation of the pattern deviation and hemifield asymmetry plots are detailed elsewhere [16].

Change in the extent of any localised sensitivity loss was determined for both perimetry conditions by counting the number of stimulus locations with statistical probability levels of $p$ less than 0.05 and comparing with baseline. The count of abnormal stimulus locations (i.e. those with a statistical significance level of $p<0.05)$ was undertaken for the complete HFA programme 10-2 field and for each quadrant of the field. In either case, only clusters of three or more abnormal stimulus locations were included in the count, that is, a conservative criterion of abnormality was used thereby minimising type I experimental error. Consequently, a cluster of three abnormal stimulus locations that crossed a mid-line would be counted in the complete field analysis but not in the quadrant analysis. For conventional perimetry, the pointwise pattern deviation plot was used to determine the extent of the localised sensitivity loss while for short-wavelength perimetry the pointwise horizontal hemifield asymmetry plot was evaluated. Global mean sensitivity (MS) was also determined for both perimetry conditions at each visit.

\section{Results}

One patient was excluded from the analysis owing to a false-positive response rate during perimetry of greater than $33 \%$. Two patients failed to attend for visit 3 and another withdrew from the study after completion of visit 3 (the resultant sample sizes were $n=23, n=21$ and $n=22$ for visits 2 (i. e. baseline), 3 (within 1 week post-treatment) and 7 (12 weeks post-treatment), respectively.

Group mean $\log$ MAR visual acuity at visit 2 was $0.076 \log$ units (SD $0.103 \log$ units); it deteriorated at visit 3 to $0.111 \log$ units (SD $0.160 \log$ units) and then recovered at visit 7 to $0.065 \log$ units (SD 0.127 $\log$ units). The change in $\log$ MAR visual acuity for each diabetic patient during the course of the study is illustrated in Figure 1.

Conventional perimetry showed an increase in the group mean number of abnormal contiguous stimulus
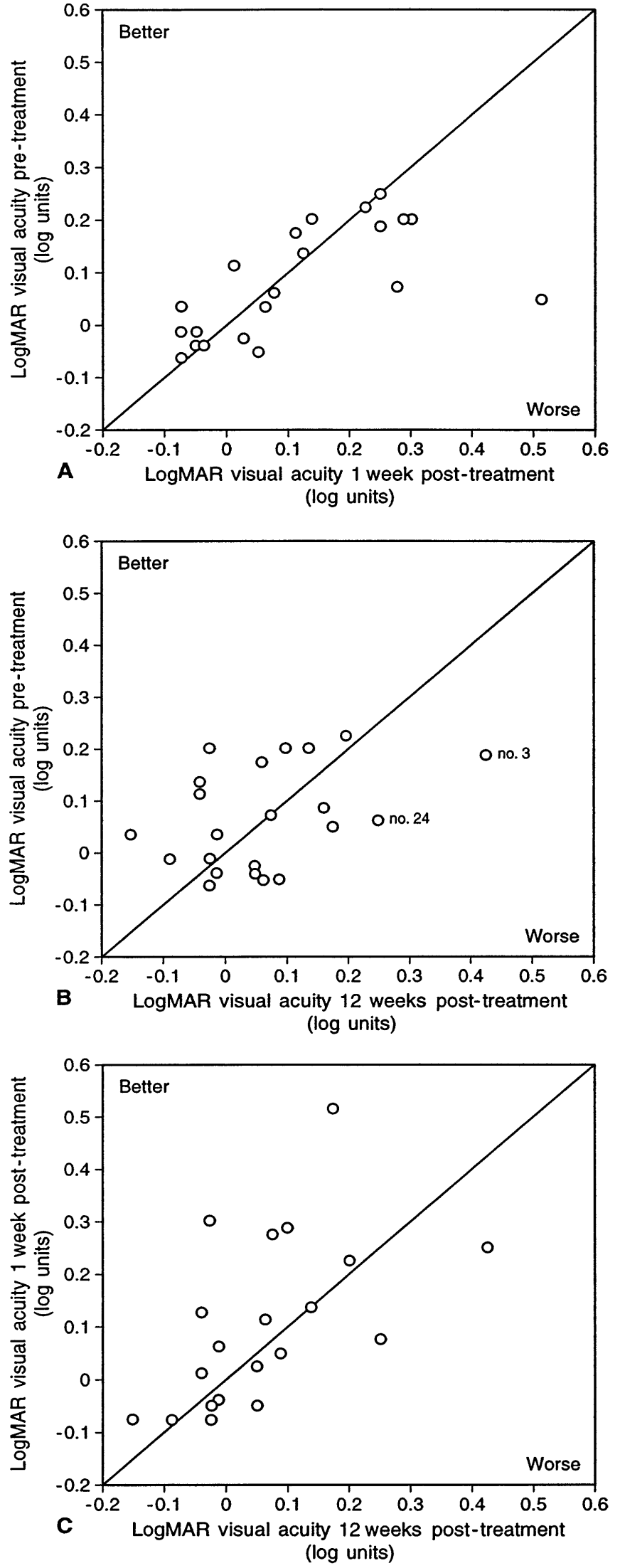

Fig. 1 A-C. Change in logMAR visual acuity over the course of the study. A. Pre-treatment vs 1 week post-treatment. B. Pretreatment vs 12 weeks post-treatment. C. 1 week post-treatment vs 12 weeks post-treatment 

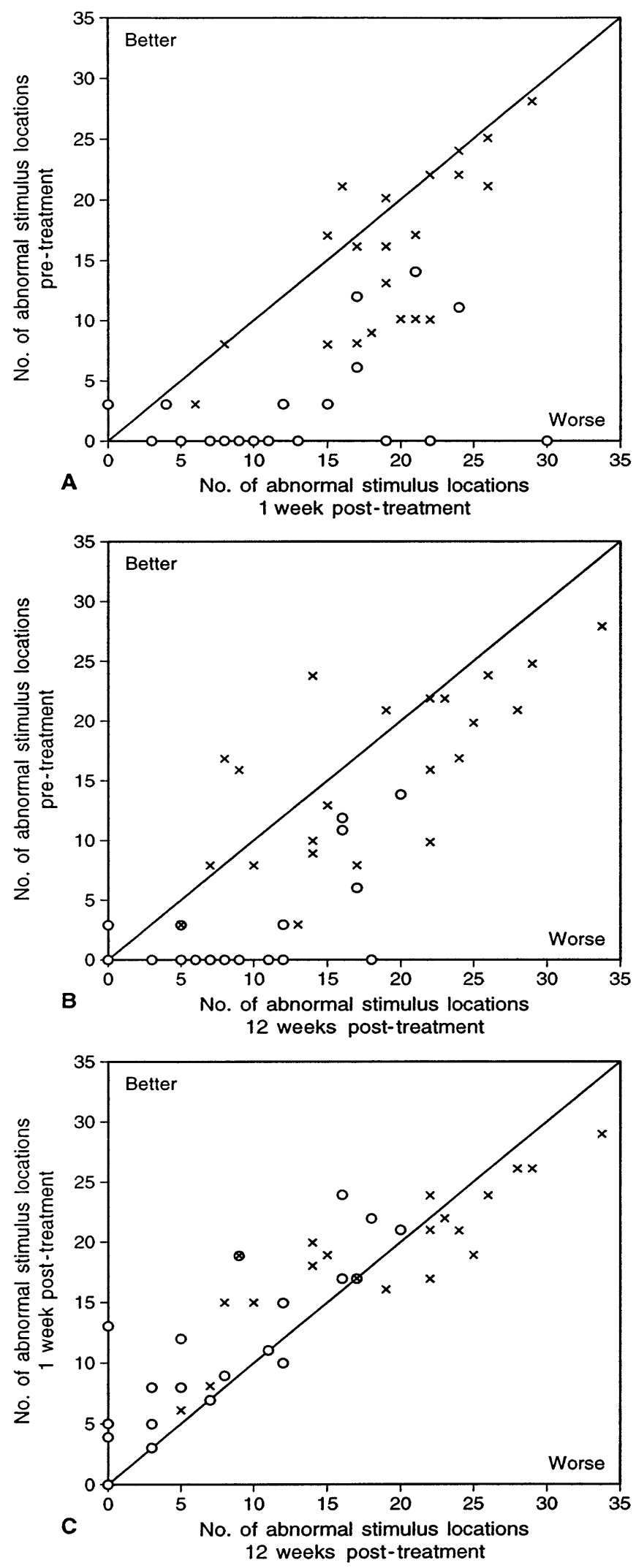

Fig. 2 A-C. Change in the number of abnormal stimulus locations (i.e. 3, or more, in any number of clusters) over the course of the study, $\bigcirc$; conventional perimetry. $\times$; short-wavelength perimetry. A. Pre-treatment vs 1 week post-treatment. B. Pre-treatment vs 12 weeks post-treatment. C. 1 week posttreatment vs 12 weeks post-treatment

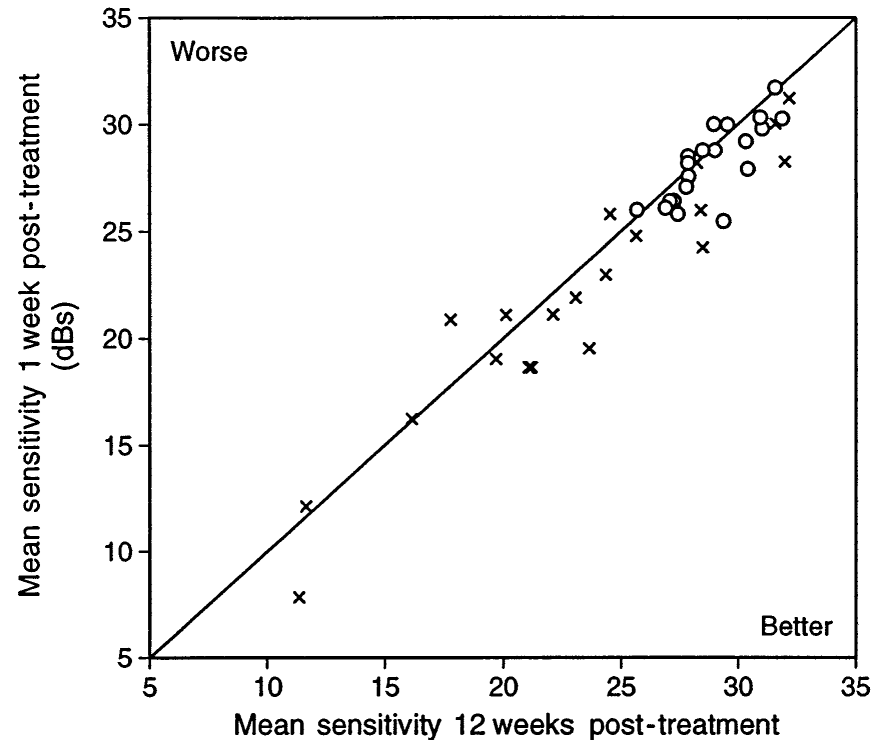

(dBs)

Fig. 3. Change in global mean sensitivity between visits 3 and 7 , $\bigcirc$; conventional perimetry. $\times$; short-wavelength perimetry. The direct comparison of mean sensitivity values between the two perimetry conditions is invalidated since the decibel scales are referenced to different maximum stimulus luminances

locations $(p<0.05)$ from 2.4 (SD 4.3, range $0-14)$ at visit 2 to 12.4 (SD 7.8, range $0-30$ ) at visit 3. At visit 7 , the group mean number of significantly abnormal contiguous stimulus locations was 8.1 (SD 6.5, range 0-20). Between visits 2 and 3, 20 patients exhibited an increase in the number of abnormal stimulus locations and 1 patient exhibited a reduction (Fig. 2). Between visits 2 and 7,18 patients exhibited an increase in the number of abnormal stimulus locations, 2 patients showed no change and 2 patients a reduction (Fig.2). Between visits 3 and 7,1 patient exhibited an increase in the number of abnormal stimulus locations, 5 patients showed no change and 14 patients a reduction (Fig. 2).

With short-wavelength perimetry, there was an increase in the group mean number of abnormal contiguous stimulus locations from 15.4 (SD 7.3, range 3-28) at visit 2 to 19.2 (SD 5.5, range 6-29) at visit 3. At visit 7, the group mean number of abnormal contiguous stimulus locations was 18.2 (SD 7.9, range 5-34). Between visits 2 and 3,15 patients exhibited an increase in the number of abnormal stimulus locations, 3 patients showed no change and 3 patients a reduction (Fig. 2). Between visits 2 and 7, 18 patients exhibited an increase in the number of abnormal stimulus locations, 1 patient showed no change and 3 patients a reduction (Fig. 2). Between visits 3 and 7, 10 patients exhibited an increase in the number of abnormal stimulus locations, 2 patients showed no change and 8 patients a reduction (Fig. 2).

Group mean global MS assessed by conventional perimetry was $29.2 \mathrm{~dB}$ (SD $1.3 \mathrm{~dB}$, median $29.3 \mathrm{~dB}$ ) 
Table 3. Summary table showing the spatial relation between area of photocoagulation and the quadrant-wise change in the number of abnormal contiguous stimulus locations for visits 3 and 7 (in parenthesis) relative to baseline. Only clusters of 3 or more abnormal contiguous stimulus locations were includ- ed in the analysis. (-; represents a decrease in the number of abnormal stimulus locations. ${ }^{\text {a }}$; withdrew from the study after visit 3. b; failed to attend for visit 3. c; excluded from the analysis due to a high false positive response rate)

\begin{tabular}{|c|c|c|c|c|c|c|c|c|c|c|c|c|}
\hline \multirow[t]{3}{*}{ Patient } & \multirow{2}{*}{\multicolumn{4}{|c|}{ Laser applied: }} & \multicolumn{8}{|c|}{ Change in no. of abnormal contiguous stimulus locations: (visit 3 [or 7] - visit 2) } \\
\hline & & & & & \multicolumn{4}{|c|}{ Conventional } & \multicolumn{4}{|c|}{ Short-wavelength } \\
\hline & $\mathrm{SN}$ & ST & IT & IN & IT & IN & $\mathrm{SN}$ & ST & IT & IN & $\mathrm{SN}$ & ST \\
\hline 1 & $X$ & & & & $8[8]$ & $0[0]$ & $0[0]$ & $0[0]$ & $1[2]$ & $5[5]$ & $0[0]$ & $0[0]$ \\
\hline 2 & & $\mathrm{X}$ & $\mathrm{X}$ & & $0[0]$ & $6[5]$ & $5[5]$ & $0[0]$ & $4[4]$ & $3[3]$ & 5 [3] & $-6[2]$ \\
\hline 3 & & $X$ & $\mathrm{X}$ & & $0[0]$ & $5[5]$ & 0 [0] & $0[0]$ & $3[0]$ & $1[0]$ & $-7[2]$ & $-3[4]$ \\
\hline 4 & & $X$ & $\mathrm{X}$ & & $0[0]$ & $6[0]$ & 13 [12] & $0[0]$ & $0[0]$ & $0[0]$ & $1[6]$ & $0[-1]$ \\
\hline $5^{\mathrm{a}}$ & $X$ & & & $X$ & $4[-]$ & $13[-]$ & $9[-]$ & $4[-]$ & $0[-]$ & $4[-]$ & $8[-]$ & $4[-]$ \\
\hline 6 & $X$ & $X$ & $\mathrm{X}$ & & $0[0]$ & $5[4]$ & $7[6]$ & $0[0]$ & $0[4]$ & $4[4]$ & 0 [1] & $-8[5]$ \\
\hline $7^{b}$ & & $X$ & & & $-[0]$ & $-[3]$ & $-[0]$ & $-[0]$ & $-[-10]$ & $-[0]$ & $-[0]$ & $-[0]$ \\
\hline 8 & & & $\mathrm{X}$ & $\mathrm{X}$ & $0[0]$ & $0[0]$ & $0[0]$ & $-3[-3]$ & $0[0]$ & $0[0]$ & $-4[-4]$ & $-1[5]$ \\
\hline 9 & $X$ & & $X$ & $\mathrm{X}$ & $4[0]$ & $0[0]$ & $0[0]$ & $0[0]$ & $0[0]$ & $0[0]$ & $0[0]$ & $-2[3]$ \\
\hline 10 & & $X$ & & & $0[0]$ & $9[8]$ & $0[0]$ & $0[0]$ & $5[0]$ & $7[4]$ & $0[0]$ & 0 [0] \\
\hline 11 & & $X$ & & & 0 [0] & 8 [2] & $0[0]$ & 0 [0] & $4[0]$ & $7[5]$ & $0[0]$ & 0 [0] \\
\hline 12 & & & $\mathrm{X}$ & $X$ & $0[0]$ & $0[0]$ & 3 [2] & 3 [2] & $0[0]$ & $0[0]$ & $-1[2]$ & $2[4]$ \\
\hline 13 & & & $\mathrm{X}$ & $X$ & $0[0]$ & $0[0]$ & $0[0]$ & $8[0]$ & $-3[-3]$ & $-3[-3]$ & $1[1]$ & $5[5]$ \\
\hline $14^{\mathrm{c}}$ & $X$ & & & & $-[-]$ & $-[-]$ & $-[-]$ & $-[-]$ & $-[-]$ & $-[-]$ & $-[-]$ & $-[-]$ \\
\hline 15 & & & $X$ & & $0[0]$ & $0[0]$ & $1[-3]$ & $0[0]$ & $0[0]$ & $0[0]$ & $4[0]$ & $-1[6]$ \\
\hline 16 & $X$ & & & $\mathrm{X}$ & 9 [7] & $3[0]$ & $0[0]$ & $5[0]$ & 8 [13] & $3[6]$ & $-3[-8]$ & $-9[9]$ \\
\hline 17 & & $X$ & X & & $0[0]$ & $0[0]$ & $6[3]$ & $0[0]$ & $0[0]$ & $0[0]$ & $0[3]$ & 0 [3] \\
\hline 18 & $X$ & $X$ & $\mathrm{X}$ & $X$ & $4[3]$ & 9 [9] & $5[3]$ & $4[3]$ & $-3[-5]$ & $9[8]$ & $0[0]$ & $5[3]$ \\
\hline 19 & $X$ & $X$ & $X$ & & $0[0]$ & $0[0]$ & $4[4]$ & $0[0]$ & $3[0]$ & $0[0]$ & $6[2]$ & $0[0]$ \\
\hline 20 & X & $X$ & & & 7 [12] & $3[0]$ & $0[0]$ & $0[0]$ & $5[5]$ & $0[0]$ & 0 [3] & $0[0]$ \\
\hline $21^{\mathrm{b}}$ & $X$ & $X$ & & $\mathrm{X}$ & $-[0]$ & $-[0]$ & $-[6]$ & $-[0]$ & $-[-3]$ & $-[0]$ & $-[3]$ & $-[6]$ \\
\hline 22 & $X$ & $X$ & & & $0[0]$ & $3[0]$ & $0[0]$ & $0[0]$ & $5[5]$ & $3[4]$ & $0[0]$ & $0[0]$ \\
\hline 23 & & $X$ & & & $0[0]$ & $7[3]$ & 0 [0] & 0 [0] & $0[0]$ & $6[4]$ & $0[0]$ & $0[0]$ \\
\hline 24 & $X$ & $X$ & $\mathrm{X}$ & & $0[0]$ & $0[3]$ & $3[0]$ & 0 [0] & $0[0]$ & $0[0]$ & $-2[-3]$ & $0[2]$ \\
\hline
\end{tabular}

at visit 2, $27.9 \mathrm{~dB}(\mathrm{SD} 2.2 \mathrm{~dB}$, median $28.1 \mathrm{~dB})$ at visit 3 and $28.9 \mathrm{~dB}$ (SD 1.7, median $28.8 \mathrm{~dB}$ ) at visit 7. For short-wavelength perimetry, group mean global MS was $23.8 \mathrm{~dB}$ (SD $5.4 \mathrm{~dB}$, median $25.3 \mathrm{~dB}$ ) at visit 2 , $21.5 \mathrm{~dB}$ (SD $5.9 \mathrm{~dB}$, median $21.2 \mathrm{~dB}$ ) at visit 3 and $23.4 \mathrm{~dB}$ (SD $5.8 \mathrm{~dB}$, median $23.9 \mathrm{~dB}$ ) at visit 7. The change in group mean global MS between visits 3 and 7 for conventional and short-wavelength perimetry is illustrated in Figure 3.

The spatial relation (assessed for each quadrant) between the area of photocoagulation and change in the number of abnormal contiguous stimulus locations for visits 3 and 7 relative to baseline is shown for conventional and short-wavelength perimetry in Table 3. In most of patients, laser photocoagulation resulted in a localised sensitivity loss in the corresponding quadrant of the visual field, particularly for conventional automated perimetry. No relation was found between the photocoagulation variables and the magnitude of perimetric change. Representative examples illustrating the effect of laser photocoagulation for clinically significant DMO on conventional and short-wavelength perimetry are shown in Figures 4 and 5.

\section{Discussion}

Despite proven benefit in the stabilisation (and occasionally the improvement) of visual acuity, the results of this study clearly show that laser photocoagulation for clinically significant DMO invariably results in a localised loss of perimetric sensitivity within $10^{\circ}$ eccentricity of the fovea (Figs. 4, 5). The complaints of such patients confirm that the preservation of visual acuity is at the expense of field loss. The spatial position of the post-treatment localised sensitivity loss corresponded with the area of retinal ablation (Table 3). Our findings seem to contradict a previous study [12] which described an overall diffuse depression of perimetric sensitivity after grid laser photocoagulation for DMO, however, that study used absolute sensitivity, rather than perimetric probability plots, to "define" change and consequently any direct comparison between the two studies is confounded.

Group mean $\log$ MAR visual acuity was largely unchanged over the course of the study. Conversely, logMAR visual acuity of individual patients showed considerable variation at visits 3 and 7 relative to baseline (Fig.1). Individual change in $\log$ MAR visual acuity at visit 3 relative to baseline varied from $-0.100 \log$ units (i.e. improvement) to $+0.462 \log$ units (i.e. deterioration) while at visit 7 the individual 
Patient: no.

Visit: 2

Eye: LE

Short-wavelength order: 2nd

Conventional total deviation

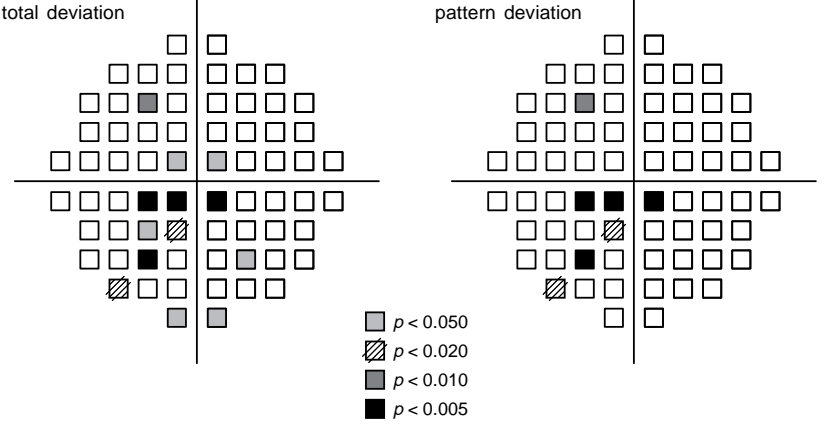

Short-wavelength

horizontal

hemifield asymmetry

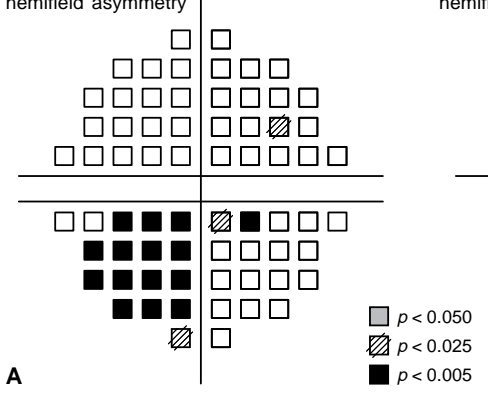

Visit: 7

Short-wavelength order: 1 st

Eye: LE
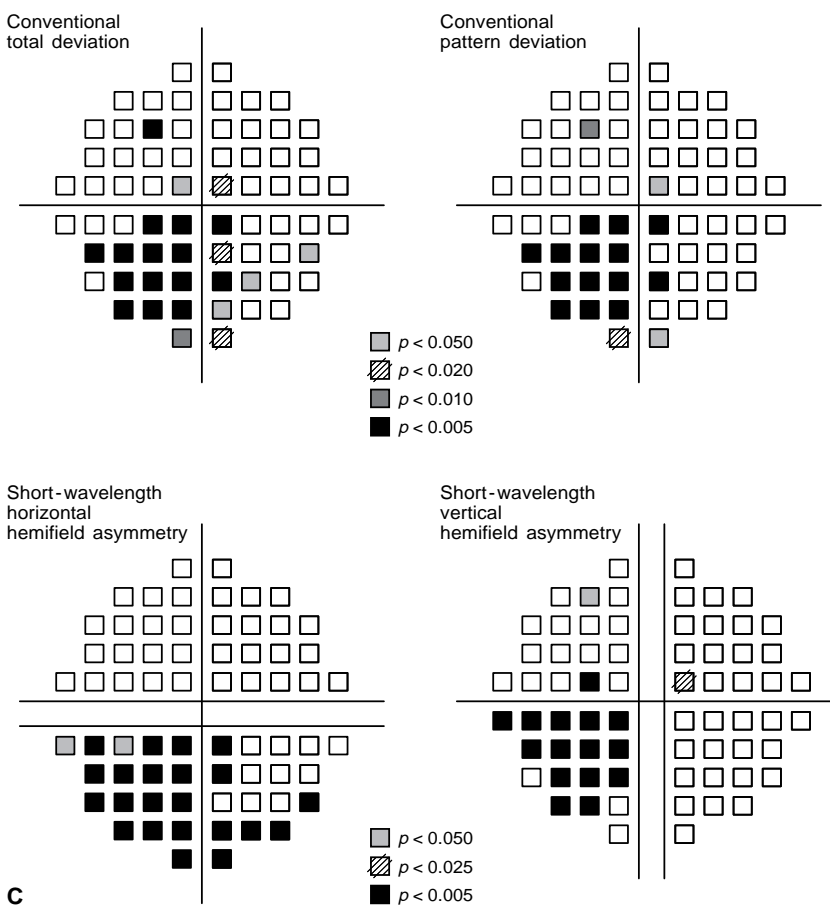

Fig. 4A-C. Automated perimetry pointwise probability plots for diabetic patient no. 1 (left eye). A visit 2. B visit 3. C visit 7. Top left; Conventional perimetry total deviation plot. Top right; Conventional perimetry pattern deviation plot. Bottom left; Short-wavelength perimetry horizontal hemifield asymmetry plot. Bottom right; Short-wavelength perimetry vertical hemifield asymmetry plot
Patient: no. 1

Visit: 3

Eye: LE
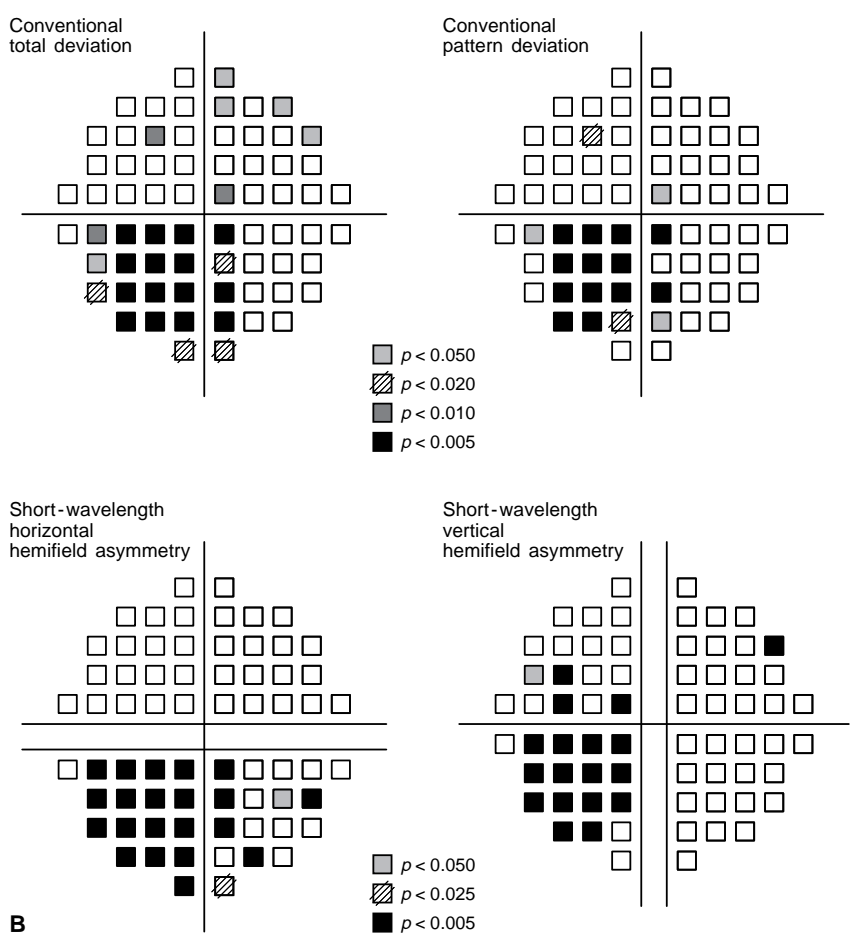

change in visual acuity relative to baseline varied from $-0.225 \log$ units to $+0.238 \log$ units. Using the $96 \%$ contrast Regan chart, any inconsistency of logMAR visual acuity greater than $\pm 0.11 \log$ units (i.e. the coefficient of repeatability, COR) is generally accepted to indicate statistically significant change [35]. In an elderly group, however, a more conservative COR value of $\pm 0.15 \mathrm{log}$ units could be more appropriate. Two patients (no.3 and no. 24) showed a deterioration in $\log$ MAR visual acuity of greater than $0.15 \log$ units at visit 7 relative to baseline (Fig.1); both patients exhibited a residual focal area of DMO involving the fovea at visit 7 .

The post-treatment increase in the extent of localised sensitivity loss was found to be greater for conventional perimetry than for short-wavelength perimetry (Fig. 2). At baseline, 22 patients exhibited a short-wavelength perimetric sensitivity loss that was of greater extent than that of conventional perimetry [16]. In addition, the extent of short-wavelength sensitivity loss was greater than the clinical area of mapped DMO in 11 patients at baseline and was approximately equal to the area of DMO in 9 patients. Consequently, laser photocoagulation was usually applied within the area of short-wavelength sensitivity loss; this resulted in a smaller post-treatment increase in the extent of the short-wavelength sensitivity loss than that of conventional perimetry. Also, the use of a size V stimulus $\left(1.724^{\circ}\right.$ angular subtense) for shortwavelength perimetry (i.e. compared with a size III 

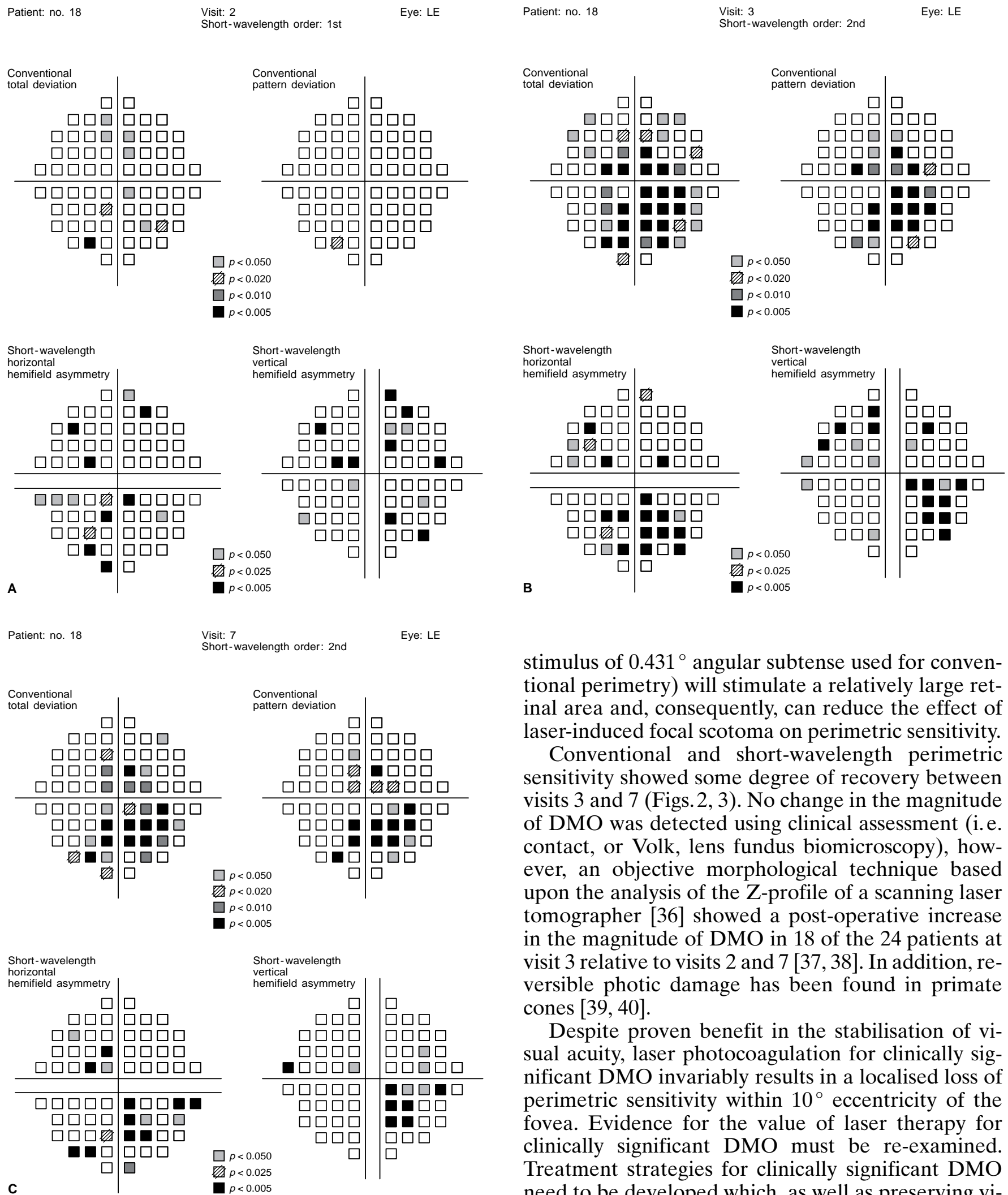

stimulus of $0.431^{\circ}$ angular subtense used for conventional perimetry) will stimulate a relatively large retinal area and, consequently, can reduce the effect of laser-induced focal scotoma on perimetric sensitivity.

Conventional and short-wavelength perimetric sensitivity showed some degree of recovery between visits 3 and 7 (Figs. 2, 3). No change in the magnitude of DMO was detected using clinical assessment (i.e. contact, or Volk, lens fundus biomicroscopy), however, an objective morphological technique based upon the analysis of the Z-profile of a scanning laser tomographer [36] showed a post-operative increase in the magnitude of DMO in 18 of the 24 patients at visit 3 relative to visits 2 and 7 [37,38]. In addition, reversible photic damage has been found in primate cones $[39,40]$.

Despite proven benefit in the stabilisation of visual acuity, laser photocoagulation for clinically significant DMO invariably results in a localised loss of perimetric sensitivity within $10^{\circ}$ eccentricity of the fovea. Evidence for the value of laser therapy for clinically significant DMO must be re-examined. Treatment strategies for clinically significant DMO need to be developed which, as well as preserving visual acuity, avoid deleterious impact on the visual field. In the meantime, perimetry should be undertaken following laser treatment for clinically significant DMO to ensure that the patient's ability to meet legal driving requirements are not compromised (this applies particularly to patients with fellow for diabetic patient no.18 (left eye). A visit 2. B visit 3. C visit 7. Top left; Conventional perimetry total deviation plot. Top right; Conventional perimetry pattern deviation plot. Bottom left; Short-wavelength perimetry horizontal hemifield asymmetry plot. Bottom right; Short-wavelength perimetry vertical hemifield asymmetry plot 
eye visual loss). Controlled treatment trials should be initiated which systematically assess the effectiveness of treatment compared with the post-treatment severity of paracentral scotomata as a function of the number, spread, power and size of laser burns. We found no relationship between the photocoagulation variables and the magnitude of perimetric change; the sample size was small, however, to show such a relation. In addition, an improved understanding of the pathogenesis of DMO is required to establish better methods of treatment, preferably pharmacological modalities.

Acknowledgements. This work was primarily funded by a postdoctoral fellowship grant from the British Diabetic Association (awarded to Dr. Hudson), also by the Manchester Royal Eye Hospital Endowments Fund (awarded to Dr. Hudson) and by a research grant from Alcon Laboratories Inc. (awarded to Drs. Flanagan and Hudson). We thank Dr Mike Patella of Humphrey Instruments for providing the short-wavelength automated perimetry normal data.

\section{References}

1. Klein R, Klein BEK, Moss SE, Davis MD, De Mets DL (1984) The Wisconsin epidemiologic study of diabetic retinopathy. IV. Diabetic macular edema. Ophthalmology 91: 1464-1474

2. Klein R, Moss SE, Klein BEK, Davis MD, De Mets DL (1989) The Wisconsin epidemiologic study of diabetic retinopathy. IX. The incidence of macular oedema. Ophthalmology 96: 1501-1510

3. British Multicentre Study Group (1983) Photocoagulation for diabetic retinopathy. A randomized controlled clinical trial using the xenon arc. Diabetes 32: 1010-1016

4. Early Treatment Diabetic Retinopathy Study Research Group (1985) Photocoagulation for diabetic macular edema. ETDRS Report Number 1. Arch Ophthalmol 103: 1796-1806

5. Early Treatment Diabetic Retinopathy Study Research Group (1991) Early photocoagulation for diabetic retinopathy. ETDRS Report Number 9. Ophthalmology 98: 766-785

6. Early Treatment Diabetic Retinopathy Study Research Group (1995) Focal photocoagulation treatment for diabetic macular oedema. ETDRS Report Number 19. Arch Ophthalmol 113: 1144-1155

7. Davies EG, Petty RG, Kohner EM (1989) Long term effectiveness of photocoagulation for diabetic maculopathy. Eye 3: 764-767

8. Early Treatment Diabetic Retinopathy Study Research group (1987) Treatment techniques and clinical guidelines for photocoagulation of diabetic macular edema. ETDRS Report Number 2. Ophthalmology 94: 761-774

9. Olk RJ (1986) Modified grid argon (blue-green) laser photocoagulation for diffuse diabetic macular edema. Ophthalmology 93: 938-950

10. Olk RJ (1990) Argon green (514 nm) versus krypton red $(647 \mathrm{~nm})$ modified grid laser photocoagulation for diffuse diabetic macular edema. Ophthalmology 97: 1101-1113

11. McNaught EI, Foulds WS, Allan D (1988) Grid photocoagulation improves reading ability in diffuse diabetic macular oedema. Eye 2: 288-296
12. Striph GG, Hart WM, Olk J (1988) Modified grid laser photocoagulation for diabetic macular edema. The effect on the central visual field. Ophthalmology 95: 1673-1679

13. Schatz HJ, Madeira D, McDonald HR, Johnson RN (1991) Progressive enlargement of laser scars following grid laser photocoagulation for diffuse diabetic macular edema. Arch Ophthalmol 109: 1549-1551

14. Guyer DR, D'Amico DJ, Smith CW (1992) Subretinal fibrosis after laser photocoagulation for diabetic macular edema. Am J Ophthalmol 113: 652-656

15. Yannuzzi LA (1990) Discussion. Ophthalmology 97: 1112-1113

16. Hudson C, Flanagan JG, Turner GS, Chen HC, Young LB, McLeod D (1998) Short-Wavelength Sensitive Visual Field Loss in Patients with Clinically Significant Diabetic Macular Oedema. Diabetologia 41: 918-928

17. Chylack LT, Wolfe JK, Singer DM et al (1993) The lens opacities classification system III. Arch Ophthalmol 111: 831-836

18. Bron AJ, Sparrow J, Brown NAP, Harding JJ, Blakytny R (1993) The lens in diabetes. Eye 7: 260-275

19. Elliott DB, Bullimore MA, Bailey IL (1991) Improving the reliability of the Pelli-Robson contrast sensitivity test. Clin Vis Sci 6: 471-475

20. Mollon JD (1982) What is odd about the short-wavelength mechanism and why is it disproportionately vulnerable to acquired damage? Report of a discussion. In: Verriest $G$ (ed) Colour Vision Deficiencies VI. Doc Ophthalmol Proc Ser 33. Dr W Junk, The Hague, pp 145-149

21. Hudson C, Wild JM, Archer-Hall JM (1993) Maximizing the dynamic range of the Humphrey Field Analyzer for blue-on-yellow perimetry. Ophthalmic Physiol Opt 13: 405-408

22. Sample PA, Johnson CA, Haegerstrom-Portnoy G, Adams AJ (1996) Optimum parameters for short-wavelength automated perimetry. Journal of Glaucoma 5: 375-383

23. Heijl A, Lindgren G, Olsson (1989) The effect of perimetric experience in normal subjects. Arch Ophthalmol 107: $81-86$

24. Hudson C, Wild JM, O’Neill EC (1994) Fatigue effects during a single session of automated static threshold perimetry. Investigative Ophthalmology and Visual Science 35: 268-280

25. Heijl A, Krakau CET (1975) An automatic static perimeter, design and pilot study. Acta Ophthalmol 53: 293-310

26. Heijl A, Lingren G, Olsson J (1987) A package for the statistical analysis of visual fields. In: Greve EL, Heijl A (eds) Seventh International Visual Field Symposium. Doc Ophthalmol Proc Ser 49. Martinus Nijhoff/Dr W Junk, Dordrecht, pp 153-168

27. Heijl A, Asman P (1989) A clinical study of perimetric probability maps. Arch Ophthalmol 107: 199-203

28. Anderson DR (1992) The single field printout with Statpac analysis. In: Kist K (ed) Automated Static Perimetry. Mosby Year Book Inc, St Louis, pp 76-90

29. Sample PA, Esterson FD, Weinreb RN, Boynton RM (1988) The ageing lens: In vivo assessment of light absorption in 84 human eyes. Invest Ophthalmol Vis Sci 29: 1306-1311

30. Sample PA, Esterson FD, Weinreb RN (1989) A practical method for obtaining an index of lens density with an automated perimeter. Invest Ophthalmol Vis Sci 30: 786-787

31. Hudson C and Wild JM (1993) The influence of pre-receptoral absorption on blue/yellow automated perimetry. In Perimetry Update 1992-93, Preceedings of the Xth International Perimetric Society Meeting. Mills RP, editor. Amsterdam, New York, Kugler Publications. 451-457 
32. Wild JM, Hudson C (1995) The attenuation of blue-on-yellow perimetry by the macular pigment. Ophthalmology 102: 911-917

33. Moss ID, Wild JM, Whitaker DJ (1995) The influence of cataract on blue-on-yellow perimetry. Invest Ophthalmol Vis Sci 36: 764-773

34. Lutze M, Bresnick GH (1991) Lenses of diabetic patients "yellow" at an accelerated rate similar to older normals. Invest Ophthalmol Vis Sci 32: 194-199

35. Elliott DB, Bullimore MA (1993) Assessing the reliability, discriminative ability, and validity of disability glare tests. Invest Ophthalmol Vis Sci 34: 108-119

36. Hudson C, Flanagan JG, Turner GS, McLeod D (1998) Scanning laser tomography Z-profile signal width as an objective index of retinal thickening due to macular oedema. Br J Ophthalmol 82: 121-130
37. Hudson C, Flanagan JG, Turner GS, Chen HC, Young LB, McLeod D (1998) Correlation of scanning laser tomography derived oedema maps with the assessment of visual function following laser photocoagulation for clinically significant diabetic macular oedema (DMO). Invest Ophthalmol Vis Sci 39 [Suppl] 467

38. Flanagan JG, Hudson C, Turner GS, Chen HC, Young LB, McLeod D (1998) Oedema map analysis of scanning laser tomography images following laser photocoagulation for clinically significant diabetic macular oedema (DMO). Invest Ophthalmol Vis Sci 39 [Suppl] 467

39. Harwerth RS, Sperling HG (1975) Effects of intense visible radiation on the increment-threshold spectral sensitivity of the rhesus monkey eye. Vision Res 15: 1193-1204

40. Sperling HG, Johnson C, Harwerth RS (1980) Differential spectral photic damage to primate cones. Vision Res 20: $1117-1125$ 